

\section{RELATED WORKS}

Durian (Durio zibethinus L) is a tropical fruit that has a special place among the local people of Southeast Asian Countries. There are 133 varieties of durian that have been officially registered with the government. According to Department of Agriculture (DOA), all of the registration name for durian species will have numerical numbers initialised with the letter D for example D24, D168 (101, Durian Ma Muar), D175 (Udang Merah), D197 (Raja kunyit, Musang King), etc., that are mainly planted all over Johor, Pahang and Penang. The price of each species is also varies.

This fruit has spiky thorny shells with round or oblong outer part and comes in a variety of size and shape. There are few ways to recognise the species of a durian for example from the colour of the husk. Some species such as D24 will have a dusty and greyish-green husk and some species such as D175 will have a yellowish-brown husk. However, some species such as D197 have both greyish-green and yellowish-brown husk colour. The other way round is by looking at the density and shape of the spikes such as D24 species may have a relatively small base of thorns and the spikes are being clustered together, D175 species may have moderately size of spikes while the spikes of D197 are far apart from each other and pyramidal shape formed from a relatively huge base of thorns. We also may observe from the shape of the husk that may come in a variety of shapes such as D24 has an oval shape and D175 has an elongated shape, while D197 has a kidney bean shape. Next, the seam of a durian such as D24 may have a circle shape that looks like a coin, D175 species has a less visible seam and D197 species has a seam like a big star or five arm starfish. Last but not least, we may categorise the species of a durian by observing the durian's crown where there is a small noticeable gap between the crown and where the spikes begin such as D197 species has a bigger gap compared to D175 and D24. These different ways to recognise durian species can be seen more clearly through Table 1 .

The work by [6] introduced a fruit recognition technique that is robust and effective to recognise fruit species. The authors used four different feature extraction techniques to represent shape, size colour and texture respectively. The authors utilised a total of thirty six images of six different types of fruit (red apples, green banana, green guava, green melon, orange, watermelon). Twenty four images are used for training purpose and twelve for testing purpose. The images of fruit were taken in different positions to get the maximum information needed for feature extraction process. The feature extraction process then extracted the area and perimeter of the fruit, determined mean value from RGB component as the colour feature, and calculated the entropy value as the texture feature. The $K$-Nearest Neighbour algorithm managed to identify the shortest distance between the feature vectors of trained dataset with feature vector of the unknown fruit. This approach managed to achieve $95 \%$ recognition accuracy but only small-size dataset is used.

Work in [7] introduced the characterisation of normal, fatty liver disease (FLD) and cirrhosis from the ultrasound images using Curvelet Transform (CT) and Entropy. This proposed method managed to achieve an accuracy of $97.33 \%$. A total of 150 ultrasound images ( 50 normal, $50 \mathrm{FLD}$, and 50 cirrhosis) were used as dataset. The pre-processing started with morphological processing operation where disk size is set to five. Then, the cropped images are resized to $500 \times 500$ using bi-cubic interpolation while Contrast-Limited Adaptive Histogram Equalization (CLAHE) is used to enhance the contrast of the ultrasound images. Next, the coefficient of CT is constructed and six various entropy features such as higher-order bi-spectrum and phase entropies ( BSE and Ph E), fuzzy entropy (f E), Kapur's entropy (k E), max entropy (maxE), Renyi's entropy (r E), Shannon's entropy (ShE), and Vajda entropy (y E) are computed to represent the statistical features of the image. The generated features are then subjected to ANOVA statistical test to obtain only highly significant ones to deliver the most important difference between the normal, FLD and cirrhosis classes. A probabilistic neural network is used to classify the three different classes. The 10-fold cross-validation is used to train and test the work and the performance is assessed. This work proposed CT instead of other transformation methods because CT performs extremely well when involving edges and other singularities along curves that plays an important roles in processing the medical images where the edges are mostly curved resultant to $97.33 \%$ of accuracies to identify normal, FLD and cirrhosis classes. The limitation of this work is that only 150 ultrasound images are used for evaluations. The performance of the method needs to be tested with larger dataset for diversity and applicability purposes.

There are a variety of fruit recognition systems that can classify different types of fruit but to our knowledge, there is only one content-based durian representation existed in the literature. The work done in [8] is designed using the global contour-based and region-based shape descriptors to extract the area, perimeter and circularity of the durians as the feature vector. The four species of durian such as D24, D101, D160 and D197 have been selected as the image dataset. A dataset of 40 images is used where 32 images used for training purposes and 8 images for testing purposes. The $K$-Nearest Neighbour is selected as the classifier to identify the durian according to the extracted feature. The 10-fold cross-validation is used for evaluation method. The proposed method managed to achieve $100 \%$ of mean accuracy in recognising the durian species. However, this work has been focusing on extracting feature from the seam of durian that might not be suitable when using for a different dataset.

Based on literature review findings, the work proposed by [8] shows that there is only a limited number of durian species at 
Table 1: The different ways to recognize a durian species

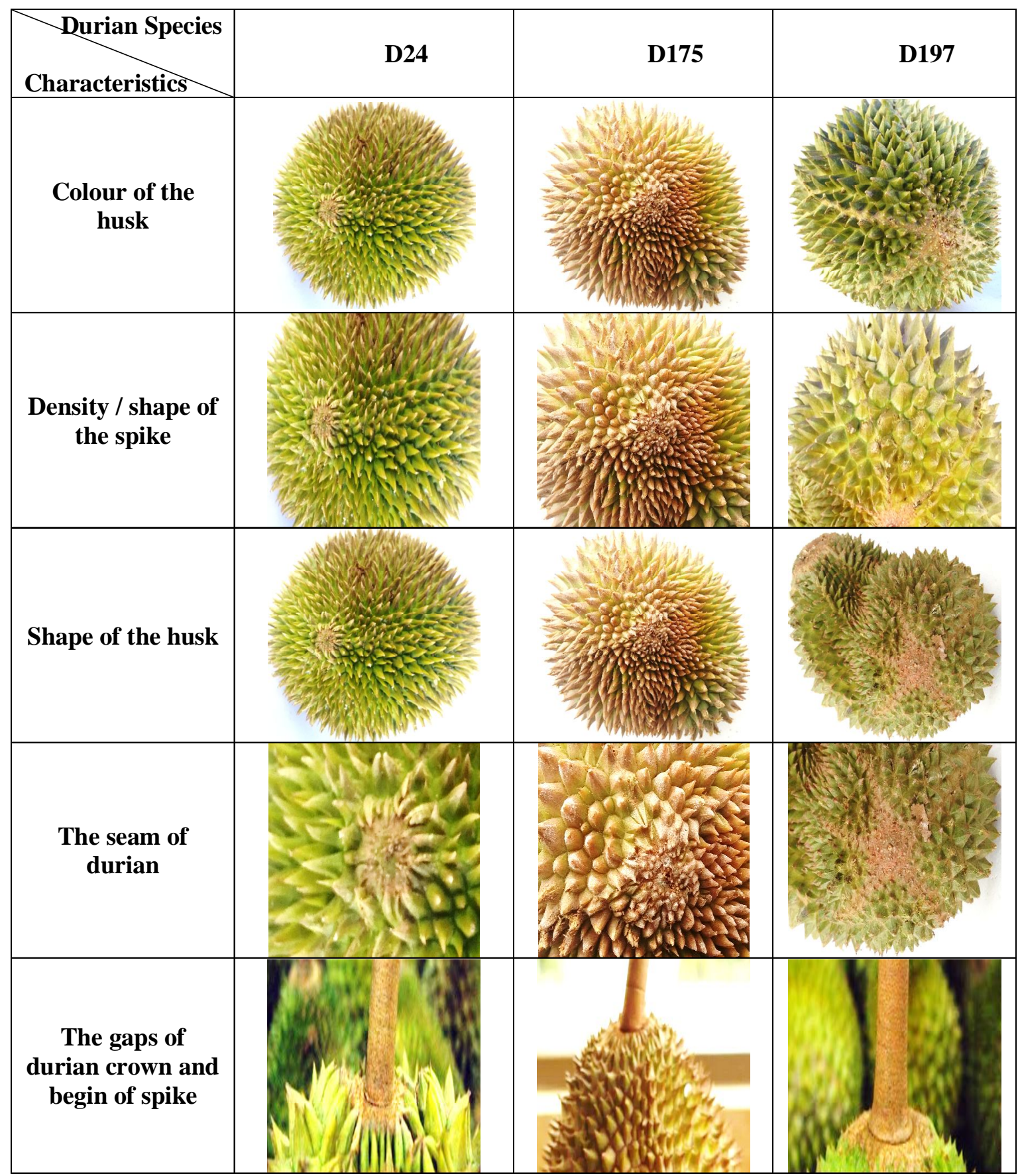

that could be recognised by focusing the feature extraction on the seam of a durian. There are 133 variety species of durian that have been registered in Department of Agriculture (DOA) [9] and the existing durian species recognition works can only classify four types of durian species. Moreover, the authors use a small size of dataset for training and testing. Despite achieving 100\% mean recognition accuracy, it has been found that the existing approach of using the Global Shape Area descriptor with fixed Region of Interest (ROI) on the seam of durian might not be suitable when using a different dataset. This would be a good method to extract feature from a visible seam such D197 that has a clear star shape but it would be a poor method for certain species such as D175 that have an invisible seam. In addition, there is also durian species that have similar seam size such as D101 and D160 that makes it harder to differentiate these species of durians. 
Thus, a study is conducted to determine other possibilities in recognising the species of a durian. This lead to the use of the durian's thorns as the main approach. The previous work of [7] has proven that the Curvelet transform works extremely well in detecting the edges and singularities along the curves that can be used as well in extracting durian's curvy thorns. Besides that, both work proposed by [6] and [8] show that $K$-Nearest Neighbour is one of the most effective classifiers for fruit-based recognition. Last but not least, the works proposed by [7] and [6] concluded that by increasing the number of dataset may increase the recognition rate for the respectively project. Hence, this has inspired the use of a larger number of dataset for this work in gaining a higher percentage of recognition rates.

\section{SYSTEM DESIGN}

Classification has two main phases, which are the training phase and testing phase. In the training phase, all of the training images will be processed where firstly the images will be read and resized to $128 \times 128$ during pre-processing. We have tested the accuracy and processing time effects of using different image sizes and the result is reported in Section 5. The species of the durian (text-based label) will also be inputted and stored where it will be utilised during training later. Next, the resized image will go through feature extraction process where Fast Discrete Curvelet Transform is computed to capture the curvilinear shape of the spikes from the durian images. Two-dimensional Fast Forward Transform (2D FFT) algorithm is performed on the resized image via wedge wrapping to gain the Fourier samples in the form of array. The norm of array will be computed from the previous Fourier samples to obtain the product of the Fourier. Then the inverse of 2D FFT is computed and resultant image's feature vector is constructed. Details on the traditional Fast Discrete Curvelet Transform can be found in the work by [10].

Next, the extracted features together with its known label (species name) will be fed into a classifier. Through an empirical study, we have tested our work on few classifiers and it has been found that $K$-Nearest Neighbour gave us the best recognition rate (result in Section 5). Out of this procedure, a trained $K$-Nearest Neighbour is generated (the predictive model), which will be intelligent enough to differentiate durian species when an unknown durian image is provided to the system as the input. The unknown durian image (testing image) prior will need to go through the same feature extraction as the training images to obtain the feature vector. The trained predictive model is used to calculate the distance between the unknown durian species with the existed durian classes. The class with the smallest distance with the unknown durian species will be assigned as the class for that durian. The proposed framework is shown in Figure 1 below and the flow chart for feature extraction and testing processes can be found in Figure 2.

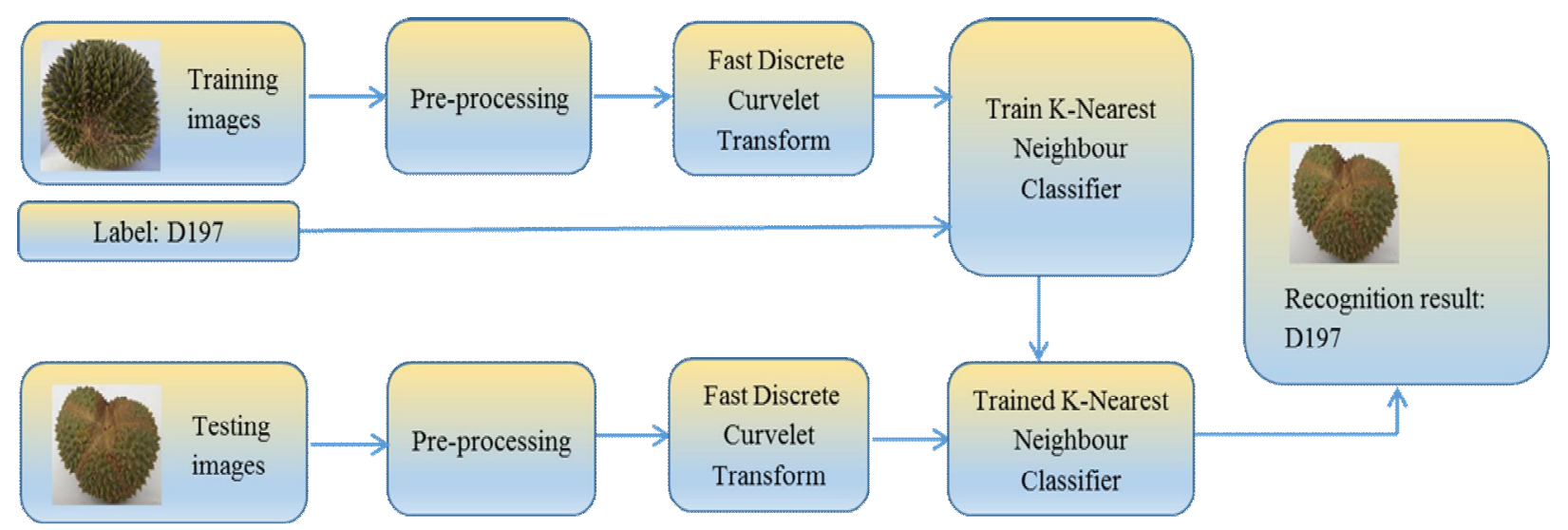

Figure 1: Overall framework of the proposed approach 


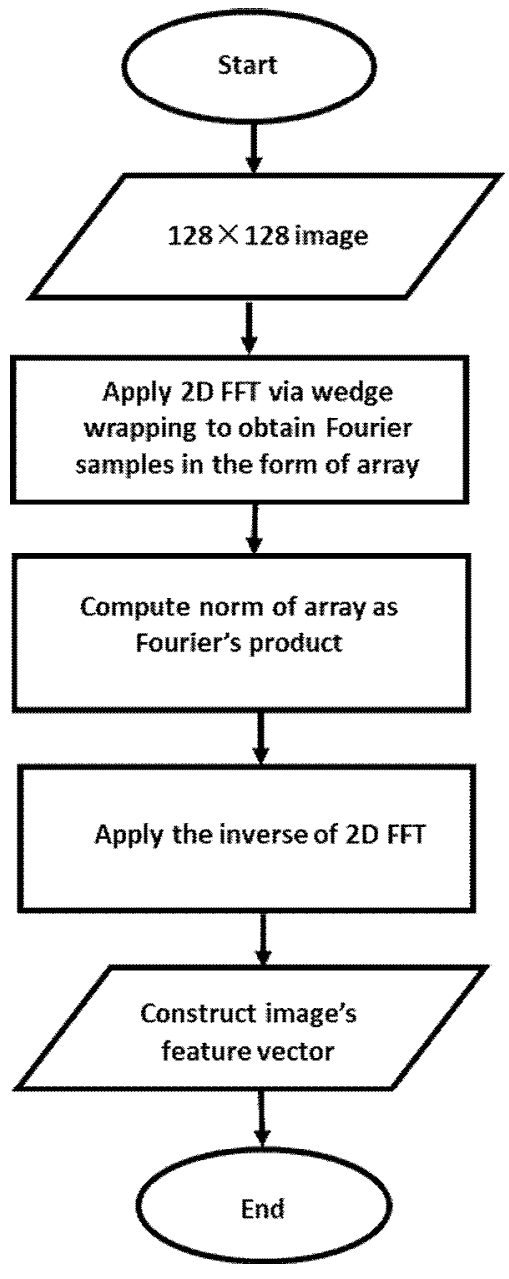

(a)

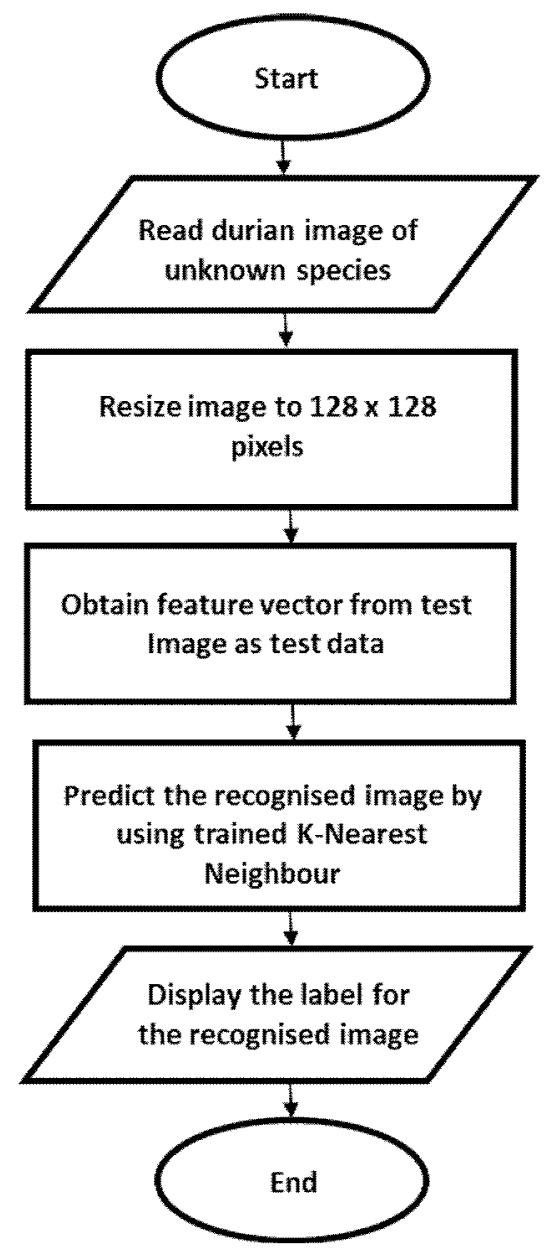

(b)

Figure 2: Process flow for (a) colour-based feature extraction (b) classification of images based on trained $K$-Nearest Neighbour

\section{SYSTEM SPECIFICATION}

HP Pavilion 15 Laptop with Windows 10 Operating System with processor of Intel® Core ${ }^{\mathrm{TM}} \mathrm{I} 5-8250 \mathrm{U}$ CPU @ $1.60 \mathrm{GHz}$ and 8 GB Random Access Memory is used to implement the work for this paper.

Figure 3 shows the Home Page for the durian species classification prototype. This Home Page contains three buttons which are the feature extraction button, training button and testing button. Users only need to click the button once to perform the desired process. The first button which is the Feature Extraction button will allow users to perform the feature extraction process. Users need to make sure the Feature Extraction button turns from grey colour into blue to select the process. Upon completing the task, the message box will pop up. The second button will allow users to perform the training process for this prototype. A message box will be popped up after completing the process. The third button in
Home page is the Testing button. It is responsible for the object recognition task. When users click the Testing button, it will direct them to another page named Recognise Species.

To identify the species of a durian, users need to upload the test image. This task can be performed by clicking the Upload Test Image button as shown in Figure 4. Another interface will pop up for users to choose the desired test image. Next, users need to click on the Recognise Image button to get the durian's species name.

\section{RESULTS AND DISCUSSION}

Six species of durian are identified and selected as the dataset. 100 durian images for each species are carefully gathered using android Vivo V3Max camera. These six durian species include D24 (Sultan), D88, D101, D160, D175 (Udang Merah) and D197 (Musang King). The dataset of durian 
species is collected during its season in approximately two months starting from beginning of July to middle of
September

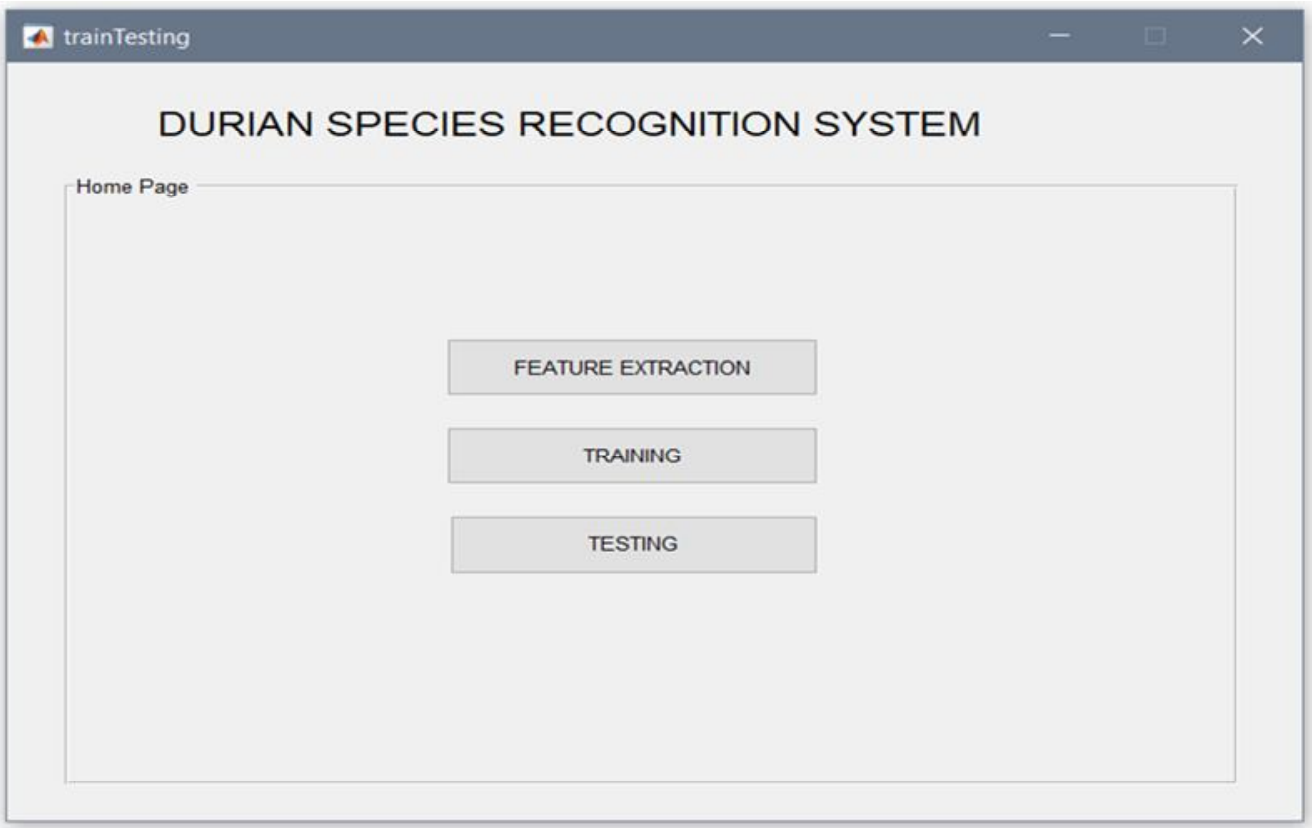

Figure 3: Home Page

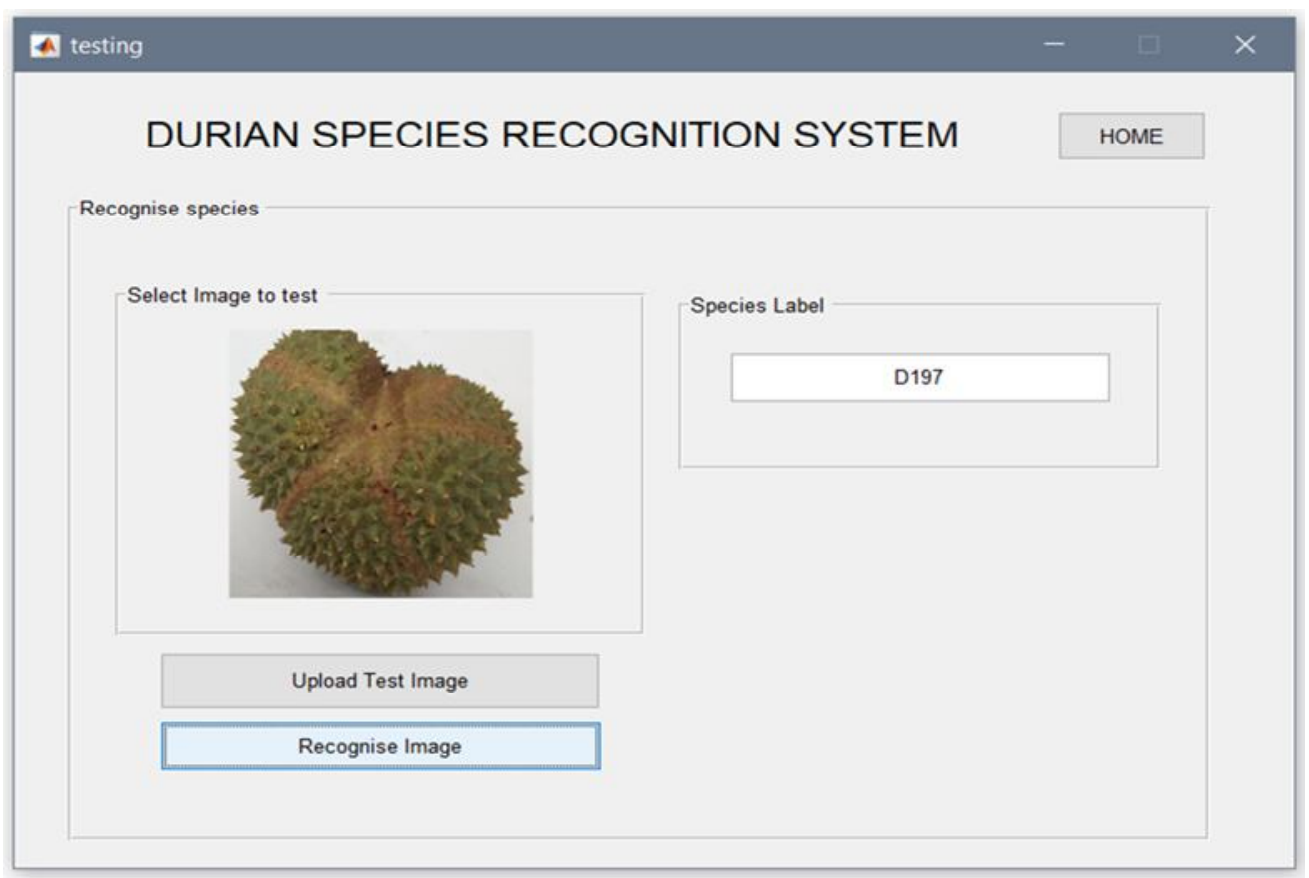

Figure 4: Display the species label

by approaching the durian sellers around Shah Alam and Sungai Buloh.

The evaluation method used for this project is 10 -fold cross validation. Several experiments were conducted for the purpose of selecting the appropriate image size, classifier type, and comparing the proposed feature extraction framework with several benchmark methods. The following sub-sections will explain more clearly about the settings used for all experiments and its result.

\subsection{Experiment to Determine the Image Size}

The experiment is conducted to choose an image size based on an optimum running time and accuracy for the proposed 
method. This is important so that even with a large amount of dataset, the running time for feature extraction process still can be relevant with a high accuracy. A total of 480 durian images from six species are used for training. Four different image sizes of $32 \times 32,64 \times 64,128 \times 128$ and $256 \times 256$ are used for the comparison. The running time is manually taken by a stop watch. The image size of $128 \times 128$ has been selected due to its optimum running time and accuracy for the proposed method in the training process.

Table 2 shows the experiment's result. The image size of 128 $\times 128$ has been selected due to its optimum running time and accuracy for the proposed method in the training process.

\subsection{Experiment to Determine the Best Classifier for the Proposed Work}

The experiment is conducted to choose the best classifier for the proposed work. This is important so that we can get the best classifier that produces the highest accuracy for object recognition process. A total of 480 durian images are used for training which include six species of durian with the same image size of $128 \times 128$. The six types of classifier include Ensemble, $K$-Nearest Neighbour, Linear Discriminant, Quadratic Discriminant, Support Vector Machine and Tree. Different framework may require different classifier to accurately classify the data set involved. This is because each data set has unique features that distinguish one from another. Hence, a different classifier may be needed by different approaches to get a relevant model fit.

Table 3 shows the experiment's result. The highest accuracy belongs to $K$-Nearest Neighbour $(92.5 \%)$ followed by Ensemble (92.3\%), Tree (82.3\%), Support Vector Machine (61.7\%), Linear Discriminant (42.3\%) and Quadratic Discriminant $(41.5 \%)$. Therefore, the $K$-Nearest Neighbour has been selected as the best classifier for the proposed work.

\subsection{Experiment on Comparing the Proposed Framework with Other Existing Approaches}

The experiment is conducted to evaluate the recognition rate between the proposed and benchmark methods. The same amount of dataset of 120 durian images will be used for testing purposes. The proposed method is compared with Global Area Shape Descriptor (Area, Perimeter, Circularity, Major Axis and Minor Axis) and Roberts Edge Detector. The recognition rate of the testing data is evaluated by 10 -fold cross validation.

Feature extraction techniques may vary depending on the suitability of the application. This is because the features to be extracted represent the relevant information that will be used for the next desired processes. Proposing the wrong feature extraction method will definitely influence and affect the recognition rate.

Table 4 shows the experiment's result. The highest accuracy for object recognition process belongs to Fast Discrete Curvelet Transform (100\%), followed by Roberts Edge Detector (81.9) and Global Area Shape Descriptor (75.5\%). The Fast Discrete Curvelet Transform is a method with superior directional representation that uses rules calculation in its formation. Besides, by using a small number of coefficient, this method able to manage the curves' discontinuities well. This is why the Fast Discrete Curvelet Transform have better edge representation and able to impersonate well the curvilinear of durians.

It has been found that the benchmark methods did not perform well for certain species like D160 and D101. This is because both of these different durian species have about the same size of durian's seam although they are not from the same species. This causes the approach to incorrectly identify the D160 as D101. Figure 5 shows the seam of durian for both species.

Table 2: Experiment to determine suitable image size

\begin{tabular}{|c|c|c|}
\hline Image Size & Running Time (Seconds) & Accuracy (\%) \\
\hline $32 \times 32$ & 74 & 89.2 \\
\hline $64 \times 64$ & 81 & 89.6 \\
\hline $128 \times 128$ & 99 & 92.5 \\
\hline $256 \times 256$ & 174 & 91.3 \\
\hline
\end{tabular}


Table 3: The experiment to determine the best classifier that will work well with the proposed feature extraction approach

\begin{tabular}{|l|c|}
\hline Type of Classifier & Accuracy (\%) \\
\hline Ensemble & 92.3 \\
\hline$K$-Nearest Neighbour & 92.5 \\
\hline Linear Discriminant & 42.3 \\
\hline Quadratic Discriminant & 41.5 \\
\hline Support Vector Machine & 61.7 \\
\hline Tree & 82.3 \\
\hline
\end{tabular}

Table 4: Accuracy of the proposed approach with benchmark methods

\begin{tabular}{|l|c|}
\hline \multicolumn{1}{|c|}{ Feature extraction method } & Accuracy (\%) \\
\hline $\begin{array}{l}\text { Global Area Shape Descriptor (Area, Perimeter, } \\
\text { Circularity, Major Axis and Minor Axis) }\end{array}$ & 75.5 \\
\hline Roberts Edge Detector & 81.9 \\
\hline Fast Discrete Curvelet Transform & 100.00 \\
\hline
\end{tabular}
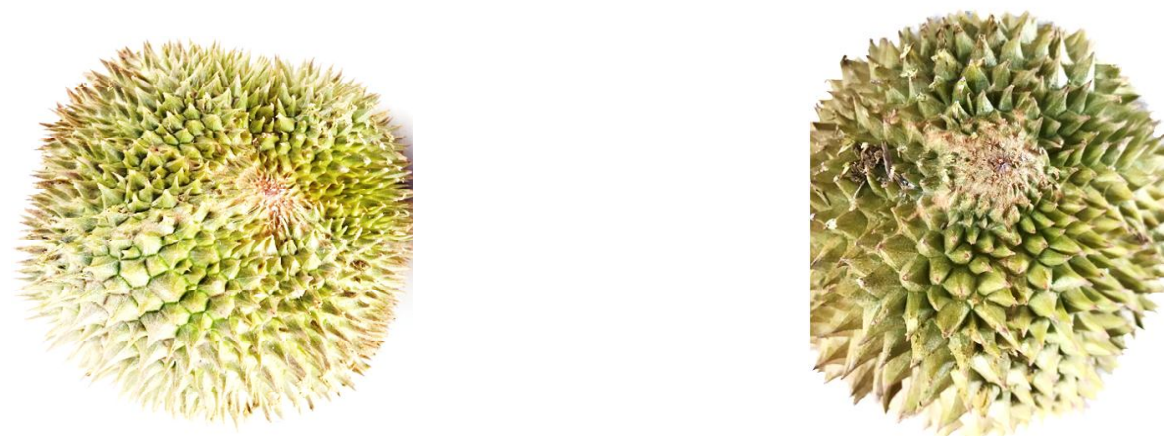

Figure 5: Durian species D101 (left) and D160 (right)

\section{CONCLUSION AND FUTURE RESEARCH}

This work proposed a durian species recognition approach based on Fast Discrete Curvelet Transform on the curvilinear shape of the durian's spikes. The extracted features have successfully differentiated durians of different species. A total of 600 images that include six species of durian (D24, D88, D101, D160, D175, and D197) have been collected and used as the dataset. Based on the developed durian species recognition prototype, the proposed feature extraction and classifier has successfully achieved a mean of $100 \%$ recognition rate based on 10 -fold cross validation.

There are still many potential future works to be executed such as expanding the dataset and add more species of durian to be recognised. Besides, a mobile version of the work would be beneficial for the public users to use it in recognising the species of durian at anytime and anywhere.

\section{ACKNOWLEDGMENT}

This work is supported by Universiti Putra Malaysia under the Putra Grant project code GP/2018/9644500.

\section{REFERENCES}

1. J. Schindelin, C. T. Rueden, M. C. Hiner, and K. W. Eliceiri. The ImageJ ecosystem: An open platform for biomedical image analysis, Molecular Reproduction and Development, vol. 82, no. 7-8, pp. 518-529, 2015. https://doi.org/10.1002/mrd.22489

2. W. C. Seng and S. H. Mirisaee. A new method for fruits recognition system, in Proc. 2009 International Conference on Electrical Engineering and Informatics, August 2009, pp. 130-134.

3. S. Hore, S. Chatterjee, S. Chakraborty, and R. K. Shaw. Analysis of different feature description algorithm in object recognition, in Computer Vision: Concepts, 
Methodologies, Tools, and Applications, 2018, pp. 601-635.

https://doi.org/10.4018/978-1-5225-5204-8.ch023

4. R. A. A. Al-falluji. Color, shape and texture based fruit recognition system, International Journal of Advanced Research in Computer Engineering \& Technology (IJARCET), vol. 5, no. 7, pp. 2108-2112, 2016.

5. N. A. Husin, S. Rahman, R. Karunakaran, and S. J. Bhore. A review on the nutritional, medicinal, molecular and genome attributes of Durian (Durio zibethinus L.), the King of fruits in Malaysia. Bioinformation, vol. 14, no. 6, pp. 265, 2018. https://doi.org/10.6026/97320630014265

6. P. Ninawe and S. Pandey. A completion on fruit recognition system using $K$-nearest neighbors algorithm, International Journal of Advanced Research in Computer Engineering \& Technology (IJARCET), vol. 3, no. 7, pp. 2352-2356, 2014.

7. U. R. Acharya, U. Raghavendra, H. Fujita, Y. Hagiwara, J. E. Koh, T. J. Hong, ..., and K. H. Ng. Automated characterization of fatty liver disease and cirrhosis using curvelet transform and entropy features extracted from ultrasound images, Computers in biology and medicine, vol. 79, pp. 250-258, 2016.

https://doi.org/10.1016/j.compbiomed.2016.10.022

8. X. Y. Nyon, M. R. Mustaffa, L. N. Abdullah, and N. A. Nasharuddin. Durian Species Recognition System Based on Global Shape Representations and $\boldsymbol{K}$-Nearest Neighbors, in Proc. 2018 Fourth International Conference on Information Retrieval and Knowledge Management (CAMP), March 2018, pp. 1-6. https://doi.org/10.1109/INFRKM.2018.8464795

9. Official Portal of Department of Agriculture of Ministry Agriculture \& Agro- Based Industry. (2019, March 22). Retrieved March 22, 2019, from http://pvpbkkt.doa.gov.my/

10. E. Candes, L. Demanet, D. Donoho, and L. Ying. Fast discrete curvelet transforms, Multiscale Modeling \& Simulation, vol. 5, no. 3, pp. 861-899, 2006.

https://doi.org/10.1137/05064182X 